\title{
Impact of birth rate, seasonality and transmission rate on minimum levels of coverage needed for rubella vaccination
}

\author{
C. J. E. METCALF ${ }^{1,2 *}$, J. LESSLER ${ }^{3}$, P. KLEPAC ${ }^{2}$, F. CUTTS ${ }^{4}$ \\ AND B. T. GRENFELL ${ }^{2,5}$ \\ ${ }^{1}$ Department of Zoology, Oxford University, Oxford, UK \\ ${ }^{2}$ Department of Ecology and Evolutionary Biology, Eno Hall, Princeton University, Princeton, NJ, USA \\ ${ }^{3}$ Department of Epidemiology, Johns Hopkins Bloomberg School of Public Health, Baltimore MD, USA \\ ${ }^{4}$ Department of Infectious Disease Epidemiology, London School of Hygiene and Tropical Medicine, \\ London, $U K$ \\ ${ }^{5}$ Fogarty International Center, National Institutes of Health, Bethesda, MD, USA
}

Received 30 November 2011; Final revision 30 December 2011; Accepted 16 January 2012; first published online 16 February 2012

\section{SUMMARY}

Childhood rubella infection in early pregnancy can lead to fetal death or congenital rubella syndrome (CRS) with multiple disabilities. Reduction of transmission via universal vaccination can prevent CRS, but inadequate coverage may increase CRS numbers by increasing the average age at infection. Consequently, many countries do not vaccinate against rubella. The World Health Organization recommends that for safe rubella vaccination, at least $80 \%$ coverage of each birth cohort should be sustained. The nonlinear relationship between CRS burden and infection dynamics has been much studied; however, how the complex interaction between epidemic and demographic dynamics affects minimum safe levels of coverage has not been quantitatively evaluated across scales necessary for a global assessment. We modelled 30-year CRS burdens across epidemiological and demographic settings, including the effect of local interruption of transmission via stochastic fadeout. Necessary minimum vaccination coverage increases markedly with birth and transmission rates, independent of amplitude of seasonal fluctuations in transmission. Susceptible build-up in older age groups following local stochastic extinction of rubella increased CRS burden, indicating that spatial context is important. In low birth-rate settings, $80 \%$ routine coverage is a conservative guideline, particularly if supplemented with campaigns and vaccination of women of childbearing age. Where birth and transmission rates are high, immunization coverage must be well above $80 \%$ and campaigns may be needed. Policy-makers should be aware of the potential negative effect of local extinction of rubella, since heterogeneity in vaccination coverage will shape extinction patterns, potentially increasing CRS burdens.

Key words: CRS, demography, extinction, rubella, seasonality, vaccine coverage.

\footnotetext{
* Author for correspondence: Dr C. J. E. Metcalf, Department of Zoology, Oxford University, Oxford, OX1 3PS, UK. (Email: charlotte.metcalf@zoo.ox.ac.uk)

The online version of this article is published within an Open Access environment subject to the conditions of the Creative Commons Attribution-NonCommercial-ShareAlike licence $<$ http://creativecommons.org/licenses/by-nc-sa/2.5/ $>$. The written permission of Cambridge University Press must be obtained for commercial re-use.
} 


\section{INTRODUCTION}

Rubella is a directly transmitted childhood infection that induces lifelong immunity. Infection in children is rarely severe. However, maternal rubella infection during the first trimester of pregnancy can lead to infection of the fetus, causing hearing impairment, cataracts and congenital heart disease which form the classical triad of congenital rubella syndrome (CRS) [1]. Other manifestations can include microcephaly, low birth weight, and mental retardation. Consequently, those with CRS often require lifelong care. Thus in high- and middle-income countries, economic benefits from elimination of CRS are estimated to be high [2].

Rubella vaccine has been licensed for nearly 50 years, is highly efficacious and available as combined measles-rubella (MR) or measles-mumps-rubella (MMR) vaccine. However, the challenge of preventing CRS by rubella-containing vaccine (RCV) is complex, because feedbacks between incidence and rate of infection mean that routine infant vaccination ('universal vaccination') will tend to increase the average age of infection, although if vaccine coverage is high enough, the age-specific incidence of rubella decreases at all ages [3]. Thus, an intervention that is not implemented optimally may actually worsen the situation.

Epidemiological models are useful tools for assessing the risks and benefits of immunization [4]. Because of the complexity of serious disease risk as a function of age and nonlinear epidemic dynamics, there is a long history of modelling rubella vaccination, especially in high-income, low birth-rate contexts [5-8]. However, these analyses do not consider a range of factors known to strongly impact nonlinear epidemic dynamics. Specifically, we do not know how a wider range of birth rates (which can strongly affect periodicity of immunizing infections [9]) will affect the relationship between infection and disease risk in rubella. Further, the impact of seasonal forcing of infection, spatial dynamics and demographic stochasticity (all of which can be profound for infections in general and rubella in particular $[10,11])$ have not been considered.

Current proposals to roll out rubella vaccination globally require us to address these dynamical gaps, and explore rubella infection and disease dynamics across a range of contexts that are globally representative. Here, we explore minimum coverage required to prevent a CRS increase after introduction of universal rubella vaccination, rooting our analysis in the current public health context, and ranging over the plausible extent of demographic contexts and remaining uncertainties in rubella transmission. The results of this analysis are also more generally relevant to any infection with a complex relationship between infection and disease risk as a function of age.

To prevent CRS, two approaches have been used. The first aims to provide direct protection to women before they reach childbearing age by selectively vaccinating girls and young women $[5,12]$. This approach has little effect on population-wide rubella transmission and has been recommended by WHO for countries aiming to reduce CRS incidence only $[13,14]$. The second approach aims to reduce or eliminate rubella transmission by universal vaccination of male and female infants and young children, sometimes combined with supplementary catch-up campaigns in older age groups [3, 14]. Previous mathematical models indicate that if vaccination of infants and young children is to be the sole strategy, coverage of at least around $80 \%$ is required $[5,7,8]$ to avoid a potential increase in the burden of CRS $[5,12,15]$ and this is the recommendation of WHO $[3,13,14]$. With sufficient immunization, control of rubella without increased CRS does appear to be tractable: the region of the Americas has sustained high infant RCV coverage and conducted mass campaigns of adolescents and adults, and reported elimination of endemic rubella transmission in 2010 [3]. However, it is not clear, that these targets can be met in all countries: in 2009, WHO-UNICEF estimates for global routine measles vaccine coverage of infants and young children were $82 \%$, with just $69 \%$ for the African region (http://apps.who.int/ immunization_monitoring/en/globalsummary/time series/tswucoveragedtp3.htm; accessed 23 December 2010; note some countries in each region have attained the goal). Mass measles campaigns, known as supplementary immunization activities (SIAs), are regularly conducted in high measles-mortality countries and many countries with elimination goals [16], and will partly compensate for lower routine coverage. However, there are concerns that there is inadequate funding to support follow-up SIAs [16]. How the presence and absence of these varied types of measles immunization activities affect the safe minimum level of added rubella vaccination is a focus of our investigation (note that while rubella elimination may be a key long-term goal, this was not addressed here). 
We focus our analyses on low-income countries, where rubella vaccination is rare [3] but estimates of CRS incidence (up to 246/100 000 live births in Africa [17]) suggest high rewards of a successful vaccination programme. Modelling has so far focused mainly on high-income countries (e.g. [5, 8], although see [15]), but several factors suggest that patterns may differ in low-income contexts [15]. First, higher birth rates will entail a lower average age of infection in the absence of vaccination. Second, rubella transmission intensity may be greater (estimates of the basic reproduction number, $R_{\mathbf{0}}$, or the number of secondary infectious persons caused by a single infectious individual in a completely susceptible population range from 6.9 to 11.8 in Addis Ababa, Ethiopia [18] compared to $2 \cdot 4-7 \cdot 8$ in Europe $[6,19])$. This will decrease the average age of infection and make it more difficult to interrupt transmission. Third, vaccination coverage may be more heterogeneous in low-income countries due to isolation of communities or inequitable access to care (including access to private sector vaccination [15]). Evidence suggests that rubella has a large critical community size or population size above which stochastic extinction does not occur [20,21], and this may combine with spatially heterogeneous vaccination uptake to allow build-up of susceptible individuals, altering CRS burdens in ways not yet explored [22]. Fourth, seasonality in transmission is little known for rubella (see [21, 22]), but is high in some low-income countries for measles, another directly transmitted airborne childhood infection. Large seasonal fluctuations in transmission can affect dynamics [23] and incidence over age [24], warranting attention in the context of CRS. Relatively lowamplitude seasonality might also matter, since rubella is thought to have lower transmission than measles, which might lead to multi-annual dynamics via an interaction between seasonality and transient dynamics due to demographic stochasticity [11, 21]. Finally, the age pattern of transmission may differ from industrialized countries, particularly as transmission in industrialized countries is shaped by school terms [9], whereas school-attendance may be lower in low-income countries.

We developed a strategic model to explore CRS dynamics after introduction of vaccination across globally representative scenarios, specifically asking: (i) when is $80 \%$ vaccine coverage of infants and young children sufficient to prevent increases in CRS incidence, and how sensitive is this to $R_{0}$, seasonality, and birth rate? (ii) how is this $80 \%$ requirement altered by the addition of typical measles control immunization strategies [regular SIA ('follow-up') campaigns or a starting ('catch-up') campaign], or vaccination of women of childbearing age? and (iii) how will stochastic dynamics affect these conclusions, and what spatial scenarios are of particular concern?

\section{MATERIALS AND METHODS}

We developed an age-structured model to quantify the effects of rubella vaccination in settings with different birth rates, basic reproduction numbers, and magnitudes of seasonal variation in transmission. The key element of the model is a matrix that at every time-step defines transition from every possible epidemiological stage (e.g. infected, susceptible, recovered) and age combination to every other epidemiological stage and age combination, following methods developed previously [25-27]. The population was stratified into 80 age groups (monthly strata up to age 4 years; yearly thereafter). We assumed that mortality was constant over age (i.e. a type II pattern, similar to that in many developing countries; altering this does not affect our qualitative results), and that the probability of survival at each age was such that the population size was constant over time (at 750000 , similar to that of Niamey, Niger) given the chosen birth rate. Each age stratum was subdivided into epidemiological groups [' maternally immune' $M$ (relevant only for the first few age groups); 'susceptible' S; 'infected' I; 'recovered' $\mathrm{R}$; and 'vaccinated' $\mathrm{V}$; taken to indicate successful vaccination] [25]. The time-step of the model was set to approximate the generation time for rubella [9] at 2 weeks, i.e. there are 24 infection generations per year (see Supplementary Information S1, available online). We assumed constant rates of movement between age strata, i.e. for age stratum $a$, the rate of ageing into the next age stratum is $u_{a}=1 /$ [(length of age group $a$ in years) $\times 24$ ]; $u$ could also be set to capture a situation of cohort ageing $[15,28]$, but given the absence of evidence for term-time forcing in many contexts of interest [23], we retained the simpler formulation. Maternal immunity for rubella is estimated to last 3-9 months $[5,18]$; accordingly, we modelled the probability of remaining in the maternal immunity group over age as an exponential decay function with a constant rate of 0.95 per month (Fig. S1a). The magnitude of $R_{0}$ combined with the population size $N$ sets the intensity of transmission with $R_{0}=\bar{\beta} N$, where $\bar{\beta}$ is average transmission. To explore seasonal forcing 
effects, the intensity of transmission was varied over the year by setting $\beta_{t}=\bar{\beta}(1+\alpha \cos (2 \pi t / 24))$ for each time step $t$ (Fig. S1b); $\alpha$ reflects the factor by which the peak and lowest value of $R_{0}$ differ from the average, and $t$ is divided by 24 , as there are 24 discrete time-steps in a year. We assumed here that seasonality affects all age groups in the same way. The probability of becoming infected is defined as

$\phi_{a}(\mathbf{n}(t))=1-\exp \left[-\sum_{j} \beta_{a, j, t} I_{j, t}^{\gamma}\right]$,

where $\beta_{a, j, t}$ is the rate of transmission between individuals in age groups $a$ and $j$ at time $t$, and $\gamma$ captures heterogeneities in mixing not directly modelled $[9,29]$ and the effects of discretization of the underlying continuous time process [30]. The probability of becoming infected can be used for both deterministic and stochastic simulations (see Supplementary Information S1 for details). Figure S2 illustrates resulting deterministic dynamics, as well as sensitivity to the chosen generation time. Supplementary Information S2 and Figure S3 provide model validation. To realistically capture routine infant and young child vaccination, we parameterized our model to reflect access to measles vaccination in Zambia (Fig. S1c) according to Lessler et al. [31]. Different levels of coverage were modelled by scaling this estimated density of vaccination over age such that total coverage achieved at age 3 years reflected the desired levels of coverage. Through interference from maternal antibodies successful vaccination is less common in the very young, hence, we further multiplied the probability of receiving vaccination by an agespecific probability of vaccination success (Fig. S1d) parameterized from Boulianne et al. [32]; their results indicate that vaccine efficacy maximally attains 0.97. We modelled vaccination of women of childbearing age assuming that access to vaccination increased gradually from age 15 years (Fig. S1e) and that coverage attained was equivalent to half the coverage attained in the routine infant and young children vaccination part of the programme, scaling appropriately, as above. There is little information from which to parameterize this part of the model, as currently routine vaccination of women, other than tetanus toxoid vaccination of pregnant women, is rare in low-income countries. For both types of vaccination, continuous probability distribution functions were converted to bi-weekly transition probabilities by the appropriate integration. We modelled starting vaccination campaigns and SIAs as campaigns lasting 2 weeks, and accessing a high proportion of the targeted age range $(90 \%)$, regardless of the degree of coverage attained by routine vaccination, i.e. SIA vaccination uptake being assumed to be independent of routine vaccination uptake.

We set transmission to be constant over age. Since there is evidence that the force of infection is lower in adults than in children in both high- [33] and low-income [24] contexts, the assumption that transmission does not vary with age leads to an overestimate in the average age of infection, and the burden of CRS. Overall, given both paucity of information, and issues of numerical tractability, the model necessarily makes a number of assumptions. These are addressed in detail in the Discussion.

\section{Exploring different vaccination strategies}

We considered the outcome of different immunization strategies set to reflect current measles immunization activities including (i) universal vaccination of infants and young children only (a method that would rarely be used alone, but serves as a baseline to be combined with other strategies); (ii) universal vaccination of infants and young children and regular SIAs ('followup campaigns') targeting all children aged between 1 and 4 at 4-year intervals, starting in the fourth year after initiation of vaccination; (iii) universal vaccination of infants and young children, regular SIAs of 1- to 4-year-olds, and a starting ('catch-up') campaign in the first year of vaccination targeting 1- to 4-year-olds; (iv) the same, but with the starting campaign targeting 1- to 14-year-olds; and (v) the same, but with additionally a campaign targeting women of childbearing age. For the latter, we expanded the model to incorporate both sexes to allow for vaccination of women only (see Supplementary Information S1). For all immunization strategies, pre-vaccination equilibrium numbers of individuals in each epidemiological and age stratum were identified numerically, and the chosen immunization strategy was implemented into a population defined by this structure.

To calculate the CRS burden, we used a pattern of fertility over age to define the risk group, i.e. pregnant women in the first 16 weeks of pregnancy (Fig. S1f, set to reflect fertility in Niger) and assumed that the probability of CRS following infection during the first 16 weeks is $c=0 \cdot 65$ [15]. Combining this value, $c$, with the numerically estimated cumulative risk of infection over the past 16 weeks, denoted $\Phi_{t}$, with 
fertility in each age group, $f_{a}$, we used our model output to calculate the number of CRS cases expected in the presence or absence of vaccination at each timestep. Their ratio defines the risk ratio [5]; values $>1$ indicate more cases in the presence than in the absence of vaccination. The exact temporal pattern of the risk ratio will depend on the balance of different key epidemiological drivers (demography, seasonality, coverage). We assessed these effects taking yearly values of the risk ratio following the start of vaccination, i.e.

$\rho_{y}=\frac{\sum_{t \in y} \sum_{a} c \Phi_{t} f_{a} S_{a, t}^{*}}{\sum_{t \in y} \sum_{a} c \Phi_{t} f_{a} S_{a, t}}$,

where * indicates the situation where the population has been vaccinated. We also tracked predicted CRS incidence (Supplementary Information S1).

\section{The range of demographic and epidemiological contexts}

We explored outcomes for birth rates ranging from 12/1000 per year (e.g. European countries, China) to $50 / 1000$ per year (Niger, at the upper end of the scale; many African countries have lower birth rates); and for $R_{0}=6$ (the average reported for rubella in Europe), $R_{0}=8$ (at the high end of the scale reported for Europe) and $R_{0}=12$ (reflecting the high end of the scale for estimates in Africa and Mexico [18, 21]). For seasonality, we explored results across a range of $\alpha$ from 0 (no seasonality) to $0 \cdot 6$ (high, as estimated in Niger for measles [23]).

\section{RESULTS}

\section{CRS burden in a deterministic setting}

Changes through time of the relative CRS burden

For each vaccination strategy, we projected the population forwards through time according to demographic and epidemiological transitions, calculated the number of CRS cases expected with or without vaccination, and took their yearly ratios for different coverage levels. For all immunization strategies, first, the relative burden of CRS decreases. Then, if coverage is low and SIAs are not undertaken, the relative burden can rise, potentially reaching values $>1$ (Fig. 1a); i.e. more CRS cases occurring in that particular year than would have in the absence of vaccination. Implementing regular SIAs leads to sporadic increases in the average age of infection (by immunizing young individuals) leading to spikes in the relative burden (Fig. 1b-d); but, overall, for equivalent routine coverage, implementing SIAs results in fewer years where the relative burden is $>1$, via more effective control of the infection. Outcomes depend on birth rate and magnitude of $R_{0}$ : for lower levels of $R_{0}$ or birth rate, low vaccination coverage levels can maintain the relative burden at $<1$ even without SIAs (Fig. S4). Results are not sensitive to the exact pattern of routine immunization.

\section{Long-term effects of vaccination on CRS burden}

Next we estimated the number of CRS cases/1000 live births after 30 years across possible coverage levels (Fig. 2, showing only routine immunization). If birth rates or $R_{0}$ are low, the burden decreases relative to the pre-vaccination burden (horizontal lines) for even small increases in coverage (Fig. 2a). However, for higher birth rates or $R_{0}$, cases/1000 live births first increase, representing an increase in average age insufficiently offset by a decrease in transmission, then decrease (Fig. 2b,c). The main goal of our analysis is to identify the minimum level of coverage that is sufficient to avoid an increase in the relative CRS burden, indicated by an arrow for each curve (Fig. 2); implementing further immunization strategies reduces this necessary coverage level. In all situations, the minimum coverage level increases with the time-horizon considered, as the average age of infection continues to increase gradually over a sustained routine vaccination programme (Fig. S5). Effects of even low vaccination coverage are most beneficial where the birth rate or $R_{0}$ are lowest, which correspond to situations where the burden of CRS is greatest in the absence of vaccination (Fig. 2).

\section{Minimum required levels of coverage}

To show effects of varying birth rates and $R_{0}$ we plotted the minimum required level of coverage (indicated by the arrow, Fig. 2) as a function of birth rate for three levels of $R_{0}$. The minimum level of coverage increases with birth rate (Fig. 3, $\mathrm{x}$-axis) and $R_{0}$ (Fig. $3 a-c$, respectively), and is reduced by implementation of SIAs (Fig. 3, coloured lines) or vaccination of women of childbearing age (Fig. 3, gold line). Seasonality had little effect (Fig. S5). 
(a)
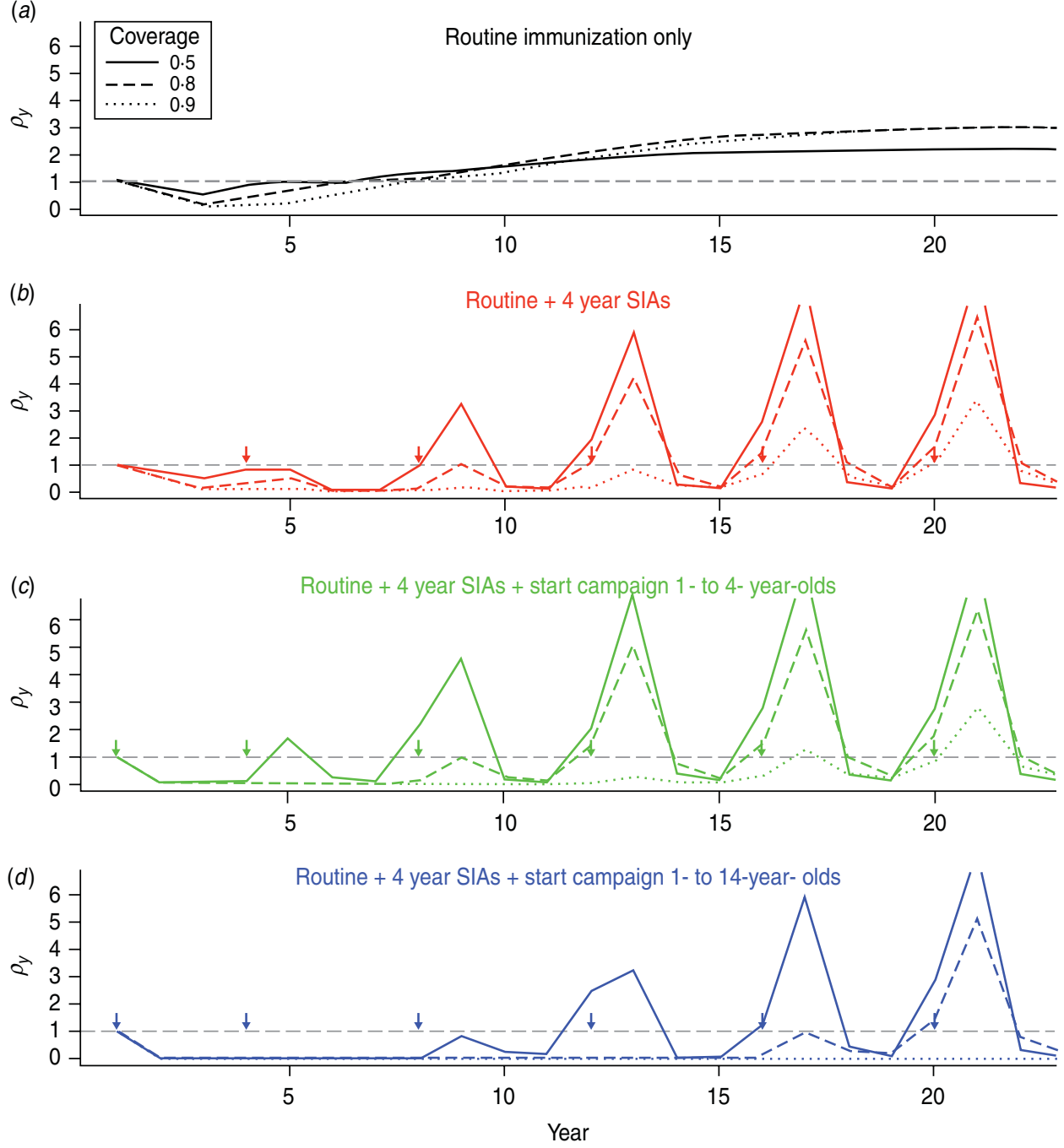

Fig. 1. Dynamics of the relative congenital rubella syndrome (CRS) burden for $R_{0}=12$, and 40 births/1000 per year for three different levels of routine vaccination coverage (see key) for four different immunization strategies (arrows indicate timing of SIAs, or starting campaigns). The x-axis shows time in years, and the y-axis indicates $\rho_{y}$, the ratio of yearly summed CRS cases in the presence and absence of vaccination. $(b-d)$ Lines corresponding to coverage higher than $50 \%$ lie close to the bottom of the plot where $\rho_{y}=0$.

\section{Requirements for implementing infant and young children immunization only}

If $R_{0}=6$, the yearly risk ratio $\rho_{y}$ remains $<1$ even for low vaccination coverage (Fig. S4a), and thus coverage levels required to retain total CRS cases below pre-vaccination levels are low (Fig. $3 a$ ). If $R_{0}=12$, after an initial 'honeymoon' period [34] during which the risk ratio $\rho_{y}$ drops (Fig. $1 a$ ), for insufficient coverage, the risk ratio may increase, i.e. more CRS cases are obtained under the vaccination programme than in the absence of vaccination; consequently, for birth rates $>20 / 1000$, coverage levels of $>80 \%$ (and nearing 100\% for birth rates of 30/1000 or higher) are required (Fig. $3 c$ ).

\section{Requirements for implementing measles-like SIAs}

Regular SIAs reduce routine coverage needed to see reductions in CRS relative to the pre-vaccination burden, since children missed by routine services may be vaccinated in the SIA (Fig. $2 a$ vs. Fig. $2 b$; Fig. 3, black vs. red lines). However, for high birth ( $>35 / 1000$ per year) and reproductive $\left(R_{0} \geqslant 12\right)$ rates, even with regular SIAs with coverage of $90 \%$, at least $80 \%$ routine vaccination coverage is required (and more if SIA coverage is lower, Fig. S6). Augmenting routine SIAs with a starting campaign extends the length of the honeymoon period [34], i.e. the time after the start of routine infant and young children vaccination before the susceptible population reaches 


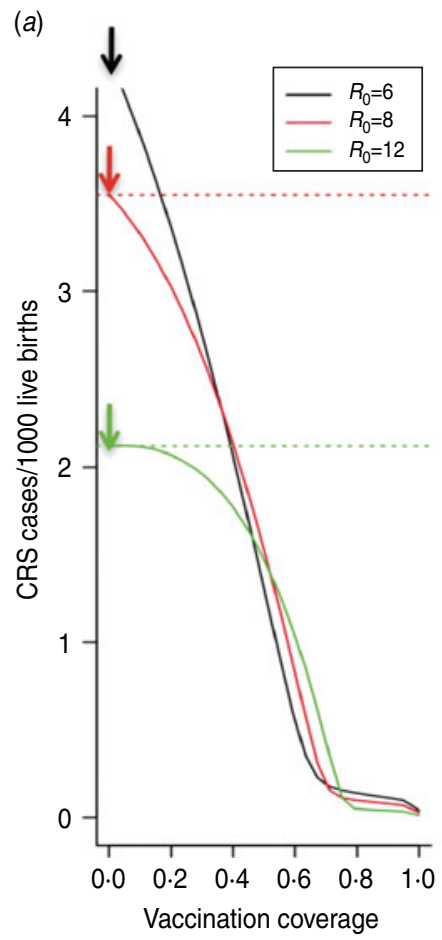

(b)

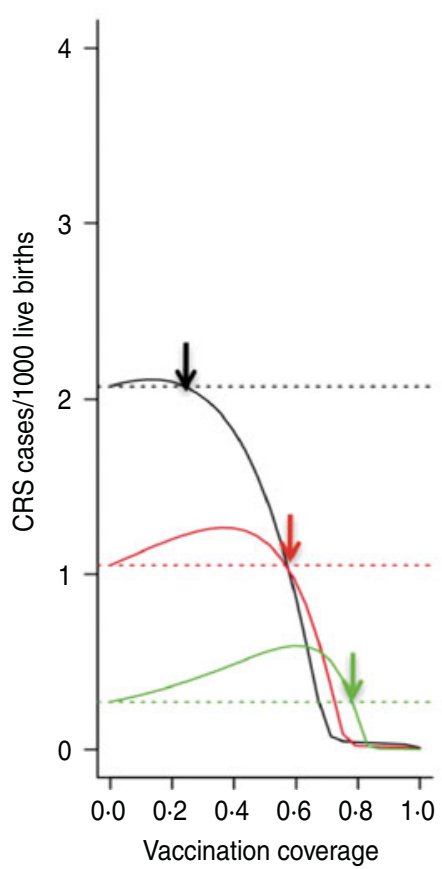

(c)

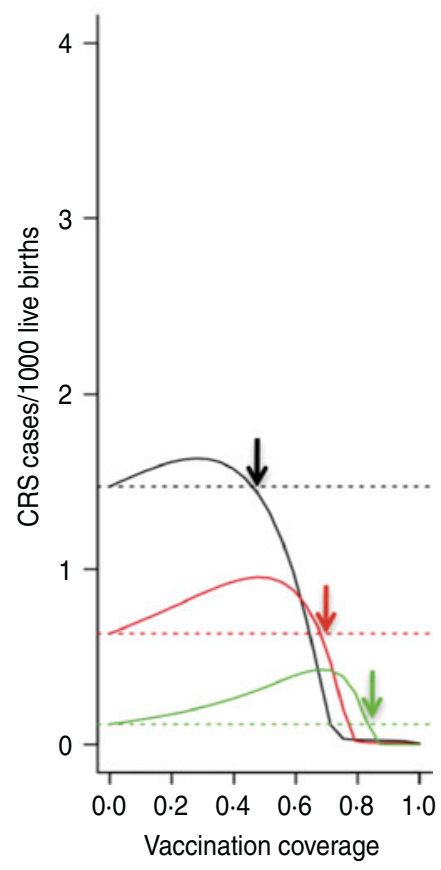

Fig. 2. Effect of routine vaccination coverage levels on congenital rubella syndrome (CRS) burden over 30 years, for different levels of routine infant and young children vaccination coverage only, total number of CRS cases/1000 live births for a population of 750000 across different birth rates for (a) 12 births/1000, (b) 30 births/1000 and (c) 40 births/1000). Three levels of $R_{0}$ are shown (in colour) under weak seasonality in transmission $(\alpha=0 \cdot 2$; higher seasonality does not alter results). Horizontal lines indicate the number of CRS cases/1000 live births occurring in the absence of vaccination; points on each curve above the corresponding line correspond to negative outcomes of vaccination; arrows indicate the level of coverage required to avoid this. Note that the exact numbers on the y-axis in any particular context will depend on the precise pattern of the fertility curve, which may itself vary with birth rate. Here, it is assumed that the pattern over age follows that for Niger.

the critical size for an epidemic (Fig. 1b-c). A greater age range in the starting campaign extends the reach of this effect (Fig. 1c-d), e.g. vaccinating children up to age 14 years $v s$. up to age 4 years means that it takes 16 years rather than only 8 years before a new birth cohort can build-up sufficiently to cause an outbreak in older children. Over time, the benefits of the starting vaccination campaign disappear as new susceptibles born after the starting vaccination campaign enter the at-risk age groups (Fig. 1b,c). Accordingly, the reduction of the minimum necessary level of vaccination to retain the burden below levels obtained in the absence of vaccination over 30 years is slight (Fig. 3 , green vs. red line). Vaccinating women of childbearing age reduces the relative burden of CRS and the minimum level of coverage required in routine programmes (Fig. 3).

\section{Spatial dynamics in a stochastic environment}

The CRS burden per 1000 live births predicted by a stochastic model that allows for local extinction/re-introduction is higher than in identically parameterized deterministic models (Fig. $4 b$ ), because local extinction of rubella leads to susceptible build-up in older age groups, increasing CRS numbers when rubella is re-introduced. Reducing imports of infected individuals, which occurs when a neighbouring location (city, or country) is vaccinated, can further substantially increase the total CRS burden (Fig. 4b), due to a greater time interval between epidemics (Fig. $4 a$; qualitative results are retained over a range of birth rates). This outcome is variable: in some simulations, rubella becomes locally extinct, and by chance, the population never experiences the arrival of an infected immigrant, so that the total CRS burden can be lower than that obtained in the absence of vaccination of the neighboring location (city or country).

\section{DISCUSSION}

Rubella is perhaps the infection for which mathematical modelling holds the greatest promise for 
(a)

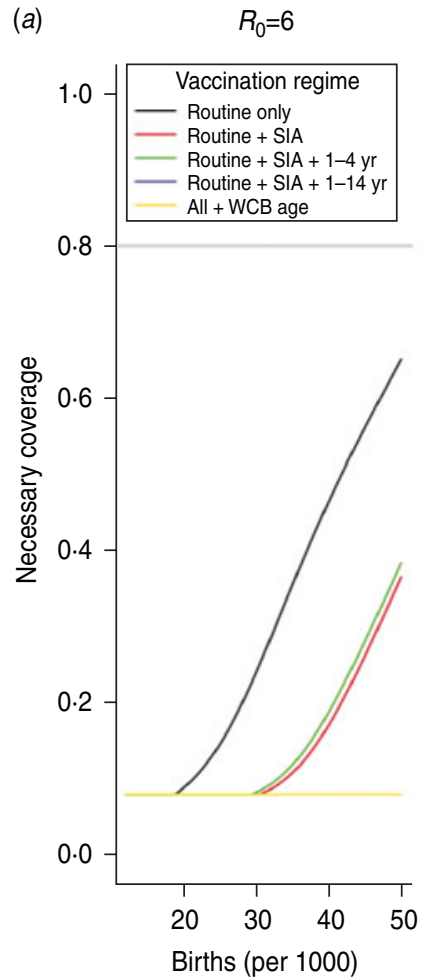

(b)

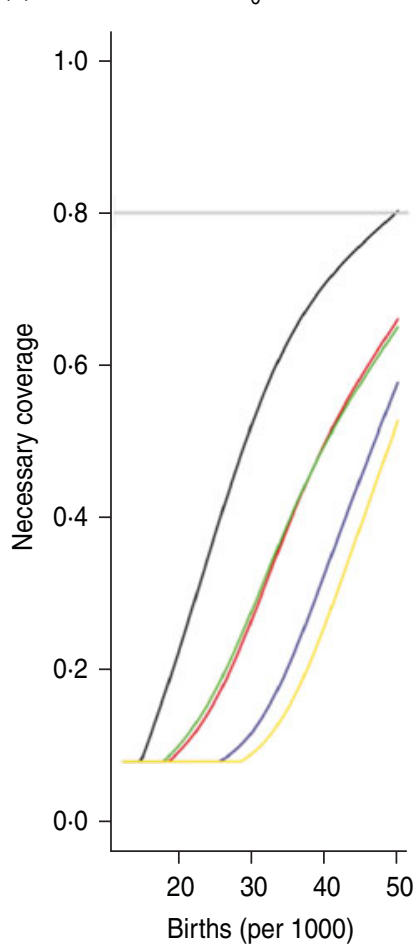

(c)

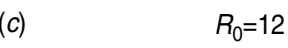

Fig. 3. Minimum level of vaccination required to retain $\rho$, the ratio of cumulative congenital rubella syndrome incidence before and after vaccination $<1$ over 30 years (y-axis), across births/1000 per year (x-axis) and levels of $R_{0}$ (panels) for five different immunization strategies, (i) routine vaccination only, (ii) routine + supplementary immunization activities (SIAs) of 1- to 4-year-olds every 4 years, (iii) routine + SIAs of 1- to 4-year-olds every 4 years + a starting campaign in 1- to 4-year-olds, (iv) routine + SIAs of 1- to 4-year-olds every 4 years + a starting campaign in 1- to 14-year-olds, and (v) routine + SIAs of 1 - to 4-year-olds every 4 years + a starting campaign in 1- to 14-year-olds + vaccination of women of childbearing age (WCB). The level currently recommended by WHO (80\%) is shown (grey line).

improving public health interventions. The epidemiology is straightforward, and thus tractable to model development, but the effect of vaccination on the burden of disease is rooted in the nonlinearities of infectious disease dynamics. These dynamics are much studied, but here we explore them in a broader demographic and spatial context than previously. Across a global spectrum of demographic and epidemiological contexts, we show that the nonlinear epidemic dynamics of rubella can interact in complex ways with both birth rates and spatial heterogeneity to influence the consequences of vaccination.

To be useful, models must be rooted a realistic public health context, and deciding whether to introduce RCVs into the routine infant immunization schedule, and what additional vaccination strategies to use requires consideration of the financial and logistical feasibility of achieving and sustaining identified minimum coverage levels in all areas of the country, and indeed in neighbouring countries. Although the burden of CRS is small compared to the overall disease burden in children in certain regions, the low cost of the rubella vaccine and the ability to deliver it as a MR combination vaccine mean that it may be one of the diseases most effectively tackled. This is particularly true where regular measles SIAs continue with high campaign coverage. Switching from the measles vaccine to the MR vaccine entails an increase in cost - in 2010 the UNICEF discounted price per dose was US\$0.8-1.50 for MMR and US $\$ 0.53$ for MR, compared to US $\$ 0.22$ for measles (http://www.unicef.org/supply/) - but no additional resources would be required for distribution: the combined vaccine would simply be substituted for the monovalent vaccine. Furthermore, a Global Alliance for Vaccines and Immunizations (GAVI) support window for the rubella vaccine is in preparation for 2012 [14].

However, rubella vaccine is also the only common vaccination whose use has the potential to lead to a negative outcome, i.e. increased incidence of CRS. We found that in the simplest analysis, where population sizes are large enough that stochastic effects can be ignored, introduction of routine infant and 
(a)

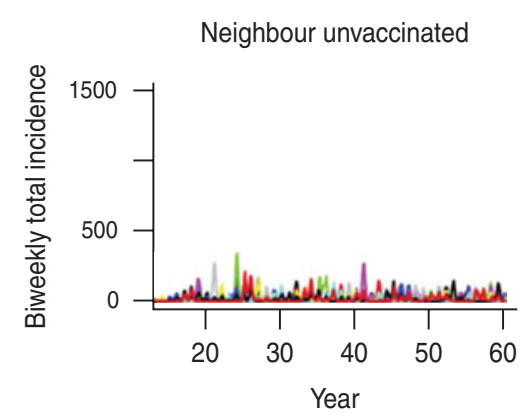

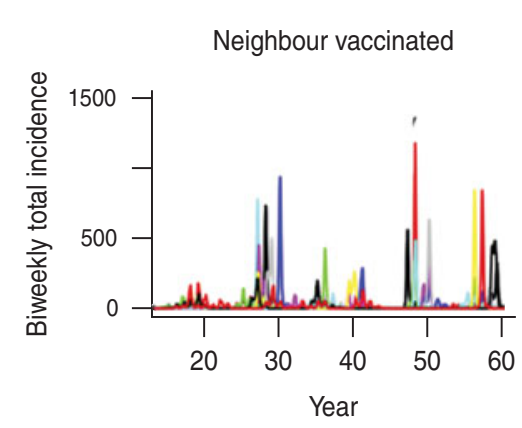

(b)

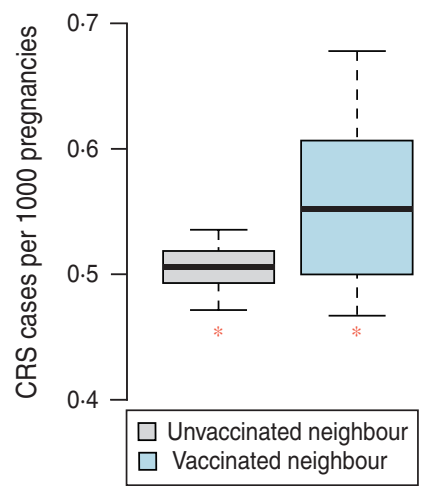

Fig. 4. The effect of reduced infected immigration on the burden of CRS in a stochastic situation. Stochastic simulations showing bi-weekly rubella incidence in the presence of some immigration of infected individuals [ $(a)$ left panel, on average three infected immigrants per year] or reduced immigration of infected individuals [ $(a)$ right panel, on average 0.3 infected immigrants per year]. Panel $(b)$ shows the numbers of CRS cases/1000 live births [medians (inner horizontal bar), $25 \%$ and $75 \%$ quantiles (box) and 0.975 and 0.025 quantiles (outer lines) across 10 simulations], and corresponding levels in the deterministic situation (red asterisks). All simulations had a birth rate of $12 / 1000$ and $R_{0}=6$ (set since the effect is expected to be strongest in communities with low transmission and/or birth rate) and population size of 50000; burdens were assessed across 40 years.

young children rubella vaccination once coverage is sustainable at $\geqslant 80 \%$ is conservative under many demographic and transmission conditions, particularly when birth rates are $<30 / 1000$ (Fig. 3), in line with previous work $[5,7,8,13,14,18]$. However, necessary vaccination coverage increases strongly with birth rate, and this coverage level is insufficient for routine vaccination alone in countries where the birth rate is $>30 / 1000$ and the basic reproductive rate of rubella is $>8$. Starting ('catch-up') campaigns followed by regular high-quality SIAs (Fig. 3) lowers the requirement for coverage across birth rates, as long as SIAs reach children who were missed by the routine programme and in the absence of stochastic effects (see below). Results are conservative relative to mixing assumptions (Fig. S5) and robust to the assumption of stable populations (Fig. S7).

Vaccination of women of childbearing age is the one strategy that will always improve the CRS situation. While such targeted campaigns should play an important role in a rubella control strategy, they face several logistical hurdles. Significantly, such a campaign could not be paired with existing measles control programmes, and thus would require a major investment in a vaccination programme aimed solely at the control of rubella. Additionally, identifying women in need of vaccination may be difficult. Overall, whether the benefits of pursuing rubella vaccination of women of childbearing age outweighs the costs will depend on coverage and the age profile of access to vaccination. In our analyses, we set a gradually increasing pattern of access to vaccination with age (Fig. S1e), and coverage equivalent to half that achieved in routine vaccination. This may be conservative: WHO reports $75 \%$ coverage for tetanus toxoid immunization, a vaccination targeted at pregnant women via routine programmes. However, vaccination of pregnant women is unlikely to be implemented for rubella and it is unclear if similar rates of success could be reached across populations.

The effect of seasonal forcing on CRS burden has not previously been addressed. Motivated by recent results highlighting both its amplitude and importance for measles dynamics in a Sahelian context [23], we explored seasonal forcing in detail here. Our results suggest that seasonality is rather negligible relative to effects of birth rate (Fig. S5). Rubella does frequently display multi-annual cycles [35], which could affect the age profile of infection [24]. Transient dynamics interacting with seasonal forcing has been proposed as the mechanism [11], rather than strong seasonal forcing per se $[10,36]$. Such effects may only shift the age of infection up by a small amount [21], and are thereby unlikely to affect the burden of CRS, but their existence (e.g. [11, 21]) implies that stochasticity is an important element of the dynamics of rubella.

If stochasticity is important, the generally positive situation for vaccination depicted by the deterministic analysis here and elsewhere $[5,35]$ needs to be 
interpreted cautiously in a world of heterogeneous vaccination rates. Local extinction might dominate rubella dynamics (e.g. see data from Peru [22] and early data from The Gambia [37]). This will allow build-up of susceptible individuals in older age groups (demonstrated for remote areas in Peru [22]) increasing the burden of CRS and potentially altering the outcomes of vaccination. Our stochastic analysis highlights that risks are high if vaccination is introduced in one location (country or community) and there is substantial cross-migration with unvaccinated communities where rubella circulation is maintained by imports. These results echo those of Vynnycky et al. [15] in that inequality in access to private-sector rubella vaccination when it is not available in the public sector may affect the CRS burden. Note that these results will also be sensitive to the assumptions of the exact generation time of the infection, as well as the value of the exponent $\gamma$ (since values closer to 1 lead to more violent dynamics more prone to extinction) and for quantitative predictions these should be carefully estimated.

Our model makes several assumptions. We assumed that a person's chance of being vaccinated in an SIA is independent of their probability of routine vaccination and vaccination in previous SIAs. However, those children not covered by routine vaccination may have been missed for some reason that increases their likelihood of being missed by SIAs, leading to a systematically unvaccinated population. Recent work has shown that this group may represent as much as $20 \%$ of the population in some countries [38]. The existence of such a group will cause us to overestimate the benefit of SIAs; hence we urge caution in the interpretation of our results and suggest that the estimated coverage of SIAs be 'discounted' and accordingly considered to be up to $20 \%$ lower if such an unreachable population is believed to exist. This is a key area for future work on specific contexts. We assumed that seasonal forcing followed a sinusoidal pattern (Fig. S1b). More realistic patterns may alter the range of multi-annual cycles [10] and could influence our conclusions on the weak effect of the magnitude of seasonal forcing on vaccination outcomes; time-series data at sub-annual time-scales would be required to investigate this. Our model is likely to be robust towards biases from other assumptions; and where biases exist, the model will tend to be conservative, overestimating rather than underestimating the minimum required coverage. For example, we assumed approximately 24 rubella generations per year; in fact there may be slightly fewer, since rubella has a generation time of about 18 days [5], implying that our model slightly overestimates yearly transmission rates, thus over-predicting minimum required levels of vaccination; sensitivity analyses suggest that this will have little impact over much of the range of vaccination (Fig. S2), but should be borne in mind for highest coverage levels. We assumed constant survival rates over age, which will result in an exponential distribution of the population over age, and may underestimate the relative proportion of women of childbearing age, and thus the CRS burden, particularly in populations where birth rates are lower (e.g. Europe). However, repeating the analysis with empirically based age trajectories of survival reflecting the likely range of age trajectories of survival which results in non-stationary populations (either growing or shrinking, depending on the balance of mortality and birth rate) does not much alter required minimum vaccination coverage (Fig. S7). Finally, we assumed that transmission is constant over age. If transmission is more clustered in younger age groups, as has been reported from surveys of contact patterns over age in European countries (Fig. S2b) the necessary vaccination coverage is higher (Fig. S8), particularly at lower birth and transmission rates (albeit retaining general patterns relative to the $80 \%$ threshold); however, the absolute number of CRS cases will be much smaller than is estimated with constant transmission over age (Fig. S9), as will be the increase driven by insufficient vaccination. Specific data needs for improved predictions relative to the various assumptions are detailed in Supplementary Information S3.

Overall, our results have several implications for introduction of RCV. First, declining birth rates in low- and middle-income countries raise the priority for rubella vaccination because; (i) falling birth rates increase the average age at infection, thus increasing the burden of CRS in the absence of vaccination, and (ii) the critical vaccination coverage required to reduce CRS incidence will be lower at lower birth rates, increasing the chances of a successful vaccination programme [36]. Second, given the current global range of birth rates, in all regions except Africa, if routine infant and young children measles vaccination coverage is $\geqslant 80 \%$ it is likely to be safe to switch to MR combined vaccines, in the absence of major heterogeneities in coverage, particularly as birth rates fall. Substantial programmatic efforts would be needed in large countries such as India; however, to 
avoid potential increases in CRS that could occur through spatial dynamic effects on transmission if the substantial heterogeneity in coverage that has been seen for measles vaccine (with up to fourfold differences in coverage between states) continued. Third, private-sector vaccination against rubella should be monitored as much as possible, to assess whether private-sector coverage has reached levels sufficient to increase CRS burdens (Fig. 2), and if so, national introduction policies should be considered. Fourth, in the African region, where birth rates remain $>40 / 1000$ in many countries and estimates of $R_{0}$ are $>6$ [18], infant and children vaccination alone should be considered with caution and SIAs would be essential as long as birth rates remain high. Even with SIAs, the potential for spikes in CRS incidence to occur in the short term implies the need for effective communication to avoid public mistrust of the programme. Fifth, the addition of vaccination of women of childbearing age has the potential to greatly enhance programme effectiveness and reduce risks of increases in CRS. Finally, the potential for local extinction and delayed outbreaks should be a factor when considering implementing any of the above recommendations. In the WHO African and SouthEast Asian regions, the only regions where universal rubella vaccination is currently uncommon [3], it would be prudent for changes in rubella vaccine policy to be taken at regional level rather than by individual countries, and for introduction of RCVs to be accompanied by intensified efforts to reduce heterogeneities in routine vaccination and SIA coverage.

The public health recommendations above are developed in the context of rubella, but the results of our analysis also provide a global overview of the degree to which complex feedback between key drivers inherent in infectious disease dynamics shape infection, and are therefore important to consider for successful control. Our broad analysis of the effects of seasonality, spatial coupling, and stochastic effects on both incidence and the age profile of incidence provide a global scale overview of the landscape of the dynamics of such infections, key for assessing safe and effective implementation of control strategies.

\section{CONCLUSIONS}

Once immunization against rubella is introduced there is no going back, so costs and benefits must be carefully weighed. For low-to-average birth-rate contexts, benefits of immunization are likely to be high across a range of levels of routine coverage, especially in the presence of additional immunization activities. For countries with high birth rates, $80 \%$ coverage of routine infant vaccination alone may not remove all risks of adverse events, and funding will need to be guaranteed for high-quality SIAs to continue in addition to routine infant and young children immunization. It is also important that policy decisions be based on not only national levels of coverage, but also their variability both sub-nationally and across regions.

\section{NOTE}

Supplementary material accompanies this paper on the Journal's website (http://journals.cambridge.org/ hyg).

\section{ACKNOWLEDGEMENTS}

S. Reef suggested analyses and provided feedback; W. J. Moss and S. A. Lowther contributed data for vaccination age profiles; E. Vynnycky gave detailed comments; the WHO Rubella Working Group provided helpful discussion. This work was funded by the Royal Society (C.J.E.M.), the Bill and Melinda Gates Foundation (C.J.E.M., B.G., J.L., P.K.), the RAPIDD program of the Science \& Technology Directorate of the Department of Homeland Security, and the Fogarty International Center National Institute of Health (B.G.) and NIH grant NIH/GM R01-GM083983-01 (C.J.E.M., B. G.).

\section{DECLARATION OF INTEREST}

None.

\section{REFERENCES}

1. Gregg NM. Congenital defects associated with maternal rubella. The Australian Hospital 1947; 14: 7-9.

2. Hinman AR, et al. Economic analyses of rubella and rubella vaccines: a global review. Bulletin of the World Health Organization 2002; 80(4).

3. World Health Organization. Rubella vaccines: WHO position paper. Weekly Epidemiological Record 2011; 86: $301-316$.

4. Anderson RM, May RM. Vaccination and herd immunity to infectious diseases. Nature $1985 ; \mathbf{3 1 8}$ : 323 329.

5. Anderson RM, May RM. Vaccination against rubella and measles: qualitative investigations of different policies. Journal of Hygiene (Cambridge) 1983; 90: $259-325$. 
6. Edmunds WJ, et al. The pre-vaccination epidemiology of measles, mumps and rubella in Europe: implications for modelling studies. Epidemiology and Infection 2000; 125: 635-650.

7. Gay N. Modeling measles, mumps, and rubella: Implications for the design of vaccination programs. Fifth International Conference on the Prevention of Infection 1998; 19: 570-573.

8. van der Heilden OG, et al. A model-based evaluation of the national immunization programme against rubella infection and congenital rubella syndrome in the Netherlands. Epidemiology and Infection 1998; 121: 653-671.

9. Bjørnstad ON, Finkenstadt B, Grenfell BT. Endemic and epidemic dynamics of measles: Estimating epidemiological scaling with a time series SIR model. Ecological Monographs 2002; 72: 169-184.

10. Keeling MJ, Rohani P, Grenfell BT. Seasonally forced disease dynamics explored as switching between attractors. Physica D 2001; 148: 317-335.

11. Bauch CT, Earn DJD. Transients and attractors in epidemics. Proceedings of the Royal Society of London, Series B 2003; 270: 1573-1578.

12. Knox EG. Strategy for rubella vaccination. International Journal of Epidemiology 1980; 9: 13-23.

13. World Health Organization. Rubella vaccine: WHO position paper. Weekly Epidemiological Record 2000; 75: 161-172.

14. World Health Organization. Meeting of the Strategic Advisory Group of Experts on Immunization, April 2011 -conclusions and recommendations. Weekly Epidemiological Record 2011; 86: 205-220.

15. Vynnycky E, Gay NJ, Cutts FT. The predicted impact of private sector MMR vaccination on the burden of congenital rubella syndrome. Vaccine 2003; 21 : 27082719 .

16. World Health Organization. Global reductions in measles mortality 2000-2008 and the risk of measles resurgence. Weekly Epidemiological Record 2009; 49: 505-516.

17. Cutts FT, Vynnycky E. Modelling the incidence of congenital rubella syndrome in developing countries. International Journal of Epidemiology 1999; 28: 1176 1184.

18. Cutts FT, et al. Sero-epidemiology of rubella in the urban population of Addis Ababa, Ethiopia. Epidemiology and Infection 2000; 124: 467-479.

19. Farrington CP, Kanaan MN, Gay NJ. Estimation of the basic reproduction number for infectious diseases from age-stratified serological survey data. Applied Statistics 2001; 50: 251-292.

20. Rios-Doria D, et al. Spatial and temporal dynamics of rubella in Peru, 1997-2006: geographic patterns, age at infection and estimation of transmissibility. In Chowell EA, ed. Mathematical and Statistical Estimation Approaches in Epidemiology. The Netherlands: Springer, 2009.

21. Metcalf CJE, et al. The epidemiology of rubella in Mexico: seasonality, stochasticity and regional variation. Epidemiology and Infection 2011; 139: 1029-1038.

22. Metcalf CJE, et al. Rubella meta-population dynamics and importance of spatial coupling to the risk of congenital rubella syndrome in Peru. Journal of the Royal Society Interface 2011 ; 8: 369-376.

23. Ferrari MJ, et al. The dynamics of measles in subSaharan Africa. Nature 2008; 451: 679-684.

24. Ferrari MJ, et al. Episodic outbreaks bias estimates of age specific force of infection: a corrected method using measles in Niamey, Niger as an example. Epidemiology and Infection 2010; 138: 108-116.

25. Klepac P, Caswell H. The stage-structured epidemic: linking disease and demography with a multi-state matrix approach. Theoretical Ecology 2010; 4: 301-319.

26. Klepac P, et al. Stage structured transmission of phocine distemper virus in the Dutch 2002 outbreak. Proceedings of the Royal Society, Series B 2009; 276: 2469-2476

27. Metcalf CJE, et al. Structured models of infectious disease: inference with discrete data. Theoretical Population Biology (in press).

28. Schenzle D. Estimation of the basic reproduction number for infectious diseases from age-stratified serological survey data. IMA Journal of Mathematics Applied in Medicine and Biology 1984; 1: 161-191.

29. Finkenstadt B, Grenfell BT. Time series modelling of childhood diseases: a dynamical systems approach. Journal of the Royal Statistical Society, Series C 2000; 49: 187-205.

30. Glass K, Xia Y, Grenfell BT. Interpreting time-series analyses for continuous-time biological models-measles as a case study. Journal of Theoretical Biology 2003; 223 : 19-25.

31. Lessler $\mathbf{J}$, et al. Maintaining high rates of measles immunization in Africa. Epidemiology and Infection 2010; 139; 1039-1049.

32. Boulianne $\mathbf{N}$, et al. Measles, mumps, and rubella antibodies in children 5-6 years after immunization: effect of vaccine type and age at vaccination. Vaccine 1995; 13: $1611-1616$.

33. Grenfell BT, Anderson RM. The estimation of agerelated rates of infection from case notifications and serological data. Journal of Hygiene (Cambridge) 1985; 95: 419-436.

34. Mclean AR, Anderson RM. Measles in developing countries part II: the predicted impact of mass vaccination. Epidemiology and Infection 1988; 100: 419-442.

35. Edmunds WJ, et al. Modelling rubella in Europe. Epidemiology and Infection 2000;125: 617-634.

36. Earn DJD, et al. A simple model for complex dynamical transitions in epidemics. Nature 2000; 287: 667-670.

37. Clarke M, et al. Epidemiological studies of rubella virus in a tropical African community. Bulletin of the World Health Organization 1980; 58: 931-935.

38. Lessler $\mathbf{J}$, et al. Measuring the performance of vaccination programs using cross-sectional surveys. PLoS Medicine 2011; 8: e1001110. 